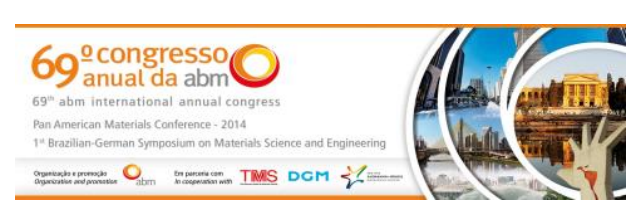

Tema: Gestão de meio ambiente e recuperação e tratamento de rejeitos

\title{
CARACTERIZAÇÃO DE PLACAS DE CIRCUITO IMPRESSO DE COMPUTADORES VISANDO RECUPERAÇÃO DE METAIS PRECIOSOS POR MEIO DE NANOPARTÍCULAS*
}

\author{
Marcos Paulo Kohler Caldas ${ }^{1}$ \\ Lucas Moraes de Sousa ${ }^{2}$ \\ Viviane Tavares de Moraes $^{3}$ \\ Denise Crocce Romano Espinosa ${ }^{4}$ \\ Jorge Alberto Soares Tenorio ${ }^{4}$
}

\section{Resumo}

O desenvolvendo da indústria de eletroeletrônicos associada ao barateamento dos produtos tem reduzido a vida útil de equipamentos levando a uma geração elevada de resíduos de equipamentos eletroeletrônicos (REEE). Um componente desse resíduo são as placas de circuito impresso, que possuem em sua composição altos teores de metais, que podem ser recuperados através de processos de reciclagem. O presente trabalho busca caracterizar placas mãe de computador, determinando sua quantidade de material orgânico, cerâmico e metálico. As placas foram direcionadas para cominuição, que foi realizada em moinho de facas com grelha de $9 \mathrm{~mm}$, seguida por um moinho de martelos, atingindo uma granulometria menor que $2 \mathrm{~mm}$. A amostra foi então quarteada, para uma maior homogeneização e passou por ensaio de digestão em água régia. $O$ licor resultante foi diluído para determinação do teor dos metais $\mathrm{Ag}, \mathrm{Al}, \mathrm{Au}, \mathrm{Ba}, \mathrm{Ca}, \mathrm{Cu}, \mathrm{Fe}, \mathrm{Ni}, \mathrm{Pb}, \mathrm{Sn}$, e $\mathrm{Zn}$ utilizando espectrometria de emissão óptica por indução de plasma (ICP-OES). O sólido filtrado, também resultante da digestão em água régia, passou por um ensaio de perda ao fogo a $800^{\circ} \mathrm{C}$ por uma hora, para determinar o teor de material orgânico. Os resultados indicaram que o $\mathrm{Cu}$ é o metal de maior concentração com $22,5 \%$ na placa de circuito impresso e que a mesma possui $43,7 \%$ de metais, $31,9 \%$ de polímeros e $24,4 \%$ de cerâmicas. A prata representa $0,04 \%$ dos metais e sua obtenção será realizada na forma de nanopartículas a partir de rotas hidrometalúrgicas.

Palavras-chave: Reciclagem; Caracterização; Nanopartículas; Metais preciosos.

\section{CHARACTERIZATION OF COMPUTERS PRINTED CIRCUIT BOARDS IN ORDER TO RECOVER PRECIOUS METAL NANOPARTICLES}

\begin{abstract}
The electronics industry developing associated with the products cheapening has reduced the equipments useful life, leading to an increase in the generation of Waste Electrical and Electronic Equipment (WEEE). The Printed Circuit Boards (PCBs) are componentes of this residue, which have large metals content in its composition that can be recovered by recycling processes. This study aims to characterize PCBs from computers to determine the amount of organic, ceramic and metallic materials. PCBs were comminuted in a cutter mill with $9 \mathrm{~mm}$ grid followed by an hammer mill, reaching a particle size less than $2 \mathrm{~mm}$. After sampling the comminuted PCBs were used in aqua regia digestion to determine the metals concentration: $\mathrm{Ag}, \mathrm{Al}, \mathrm{Au}, \mathrm{Ba}, \mathrm{Ca}, \mathrm{Cu}, \mathrm{Fe}$, $\mathrm{Ni}, \mathrm{Pb}, \mathrm{Sn}$ and $\mathrm{Zn}$. So, the aqua regia liquor was diluted and the metals content were determined using an optical emission spectrometry with inductively coupled plasma (ICP-OES) and, the filtered solid underwent a loss on ignition test at $800^{\circ} \mathrm{C}$ during one hour to determine the organic material content. The results indicate that $\mathrm{Cu}$ is the metal with the highest concentration in the studied PCB (22.5\%w.t.). This PCB has $43.7 \%$ w.t. of metals, $31.9 \%$ w.t. of polymers and $24.4 \%$ w.t. of ceramicas. The Ag concentration represents $0.04 \%$ w.t. of the metals and its recovering will occur in nanoparticles form by hydrometallurgical processes.
\end{abstract}

Keywords: Recycling; Characterization; Nanoparticles; Precious metals.

\footnotetext{
Doutorando, Eng. Química, Escola Politécnica, USP, São Paulo, SP, Brasil. Graduando, Tecnologia Ambiental, SENAI Mario Amato, São Bernardo do Campo, SP, Brasil.

Doutora, Eng. Metalúrgica e de Materiais, Escola Politécnica, USP, São Paulo, SP, Brasil

Professor(a), Dept. Eng. Química, Escola Politécnica, USP, São Paulo, SP, Brasil.
}

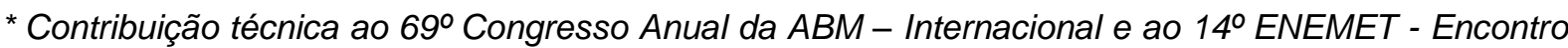
Nacional de Estudantes de Engenharia Metalúrgica, de Materiais e de Minas, 21 a 25 de julho de 2014, São Paulo, SP, Brasil.
} 


\section{INTRODUÇÃO}

A injeção de tecnologias cada vez mais avançadas no mercado faz com que aparelhos eletroeletrônicos se tornem descartáveis em períodos de tempo cada vez mais curtos. Em 2006, foram fabricados, aproximadamente, 34 milhões de televisores e 24 milhões de aparelhos de comunicação portátil, como celulares e pagers, nos E.U.A. [1]. No panorama econômico e desempenho setorial divulgado pela Associação Brasileira da Indústria Elétrica e Eletrônica, ABINEE [2], que apresenta as estatísticas do setor eletroeletrônico de 2003 a 2010, a quantidade de computadores vendidos teve um aumento de $437,5 \%$. Segundo Rao [3], o tempo médio de vida útil de um computador é de 2 a 5 anos, após isso são descartados devido a defeitos ou por se tornarem obsoletos.

Em 2011 a geração de resíduo eletroeletrônico (REEE) no Brasil foi de 678.960t com previsão de acumulo de 22,4 milhões de toneladas entre 2001 e 2030 Ribeiro [4]. As placas de circuito impresso (PCls) representam $3 \%$ de todo resíduo eletroeletrônico gerado [5], assim pode-se estimar uma geração anual de 20.369 t de PCls que serão descartadas.

As PCls são encontradas em praticamente todos equipamentos eletroeletrônicos sendo compostas de metais, polímeros e cerâmicos. As PCls de computadores pessoais, como todo REEE, são compostas basicamente de $30 \%$ de cerâmicos, $30 \%$ de plásticos e $40 \%$ de metais [6]. Em sua composição pode ser encontrada ainda metais preciosos como prata, ouro e paládio $[7,8]$ e contaminantes como 0 chumbo, que, somado ao grave problema ambiental de uma má destinação do resíduo, torna a reciclagem sua melhor alternativa de tratamento. Existem muitos estudos para reciclagem de materiais através de processos químicos [9], térmicos [10] e mecânicos [11].

Os processos químicos se baseam na transferência de metais, originalmente presentes em REEE ou em minérios, para uma solução através de extração sólidolíquido. Esta extração também conhecida como processo de lixiviação utiliza-se como agentes lixiviantes soluções ácidas, alcalinas e salinas[12]. O processo de digestão em água régia consiste do contato direto de um sólido contendo metais, com uma mistura de ácido clorídrico com ácido nítrico concentrados na proporção de $3: 1$ [13].

A moagem das PCls, etapa que antecede o processo de digestão em água régia, torna-se um procedimento importante, pois possibilita a liberação de materiais presentes no resíduo [14] além de aumentar a área da superfície de contato do sólido que passará pelo processo de lixiviação.

$\mathrm{O}$ alto custo dos processos de lixiviação associado a dificuldade na seletividade de separação dos metais pode inviabilizar economicamente o processo de reciclagem de placas de circuito impresso. A reciclagem de metais preciosos na forma de nanopartículas torna-se uma alternativa viável devido ao valor agregado e as várias aplicações de nanopartículas atualmente. Nanopartículas de prata, por exemplo, possuem aplicações em áreas variadas, podendo ser utilizados como aditivo para materiais poliméricos, materiais adsorventes de gazes ou metais e ainda em aplicações biotecnológicas [15].

O objetivo deste trabalho foi fazer a caracterização de PCls de computadores obsoletos identificando a quantidade de metais presentes em sua composição visando a recuperação de metais preciosos por meio de nanopartículas.

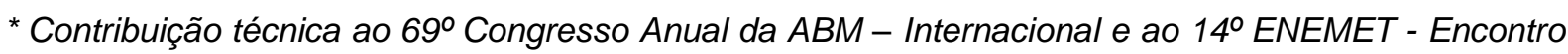
Nacional de Estudantes de Engenharia Metalúrgica, de Materiais e de Minas, 21 a 25 de julho de 2014, São Paulo, SP, Brasil.
} 


\section{MATERIAIS E MÉTODOS}

As PCls utilizadas foram cedidas pelo Centro de Descarte e Reuso de Resíduos de Informática da Universidade de São Paulo (CEDIR/USP), conforme Figura 1. Foram selecionadas placas lead free devido a substituição do chumbo por compostos de prata. Peças removíveis de maior tamanho como dissipadores de alumínio e componentes de refrigeração foram retirados manualmente.

$\mathrm{Na}$ sequencia as PCls foram: cominuidas; caracterizadas por digestão em água régia, ICP-OES e perda ao fogo.

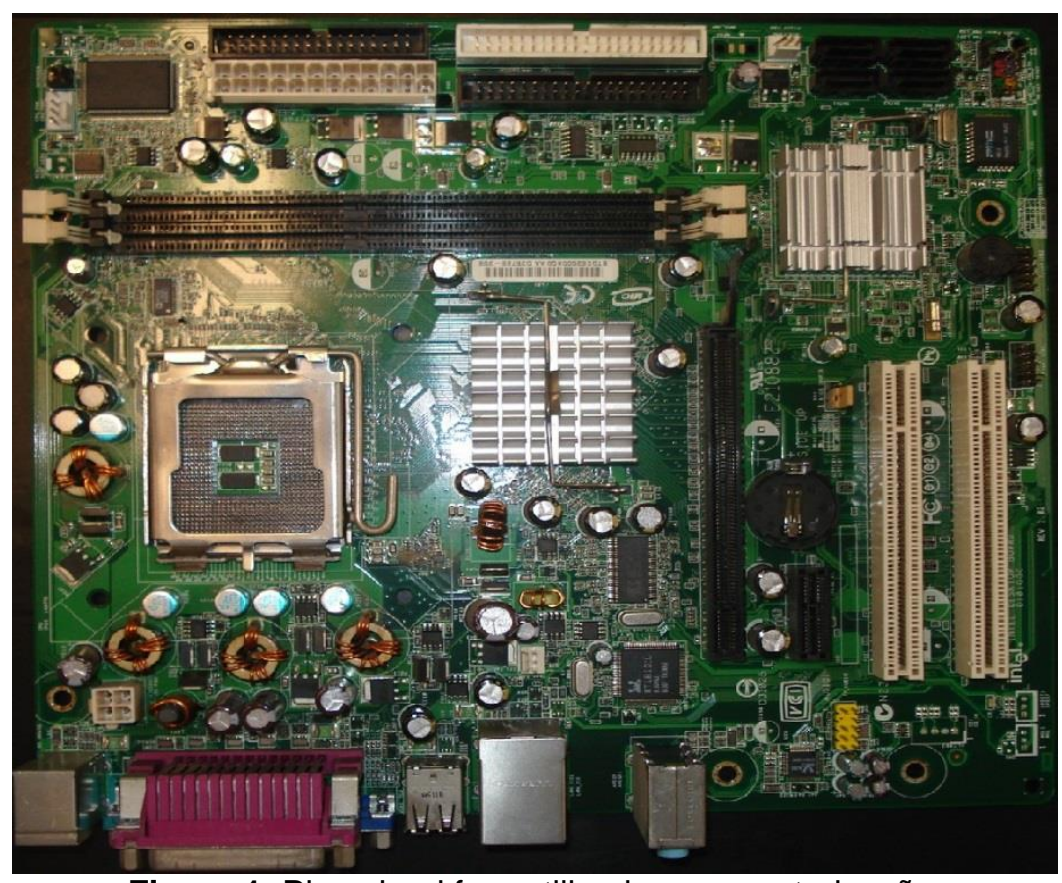

Figura 1. Placa lead free utilizada na caracterização.

\subsection{Cominuição}

Para redução do tamanho das PCls e facilitar sua moagem utilizou-se uma guilhotina manual para cortar as placas em quatro partes. Em seguida as PCls foram cominuidas em duas etapas, inicialmente passaram por um moinho de facas modelo RONE FA2305 com grelha de $9 \mathrm{~mm}$ e depois por um moinho de martelos modelo MDM 18/18 da marca Astecma com grelha de $2 \mathrm{~mm}$. Após as etapas de cominuição das PCls, o material moído foi quarteado visando melhor homogeneização das amostras.

\subsection{Caracterização}

\subsubsection{Digestão em água régia}

Uma fração quarteada de $20 \mathrm{~g}$ foi solubilizada pelo contato direto com uma solução de água régia na relação de 3:1 $\left(\mathrm{HCl}: \mathrm{HNO}_{3}\right)$ na proporção sólido líquido de 1:20 [13] (1 g da alíquota para $20 \mathrm{ml}$ de solução de água régia), portanto $20 \mathrm{~g}$ da amostra foi colocada em contato direto com $400 \mathrm{ml}$ de solução ácida.

A solubilização dos metais em água régia foi feita durante 24 horas a temperatura ambiente.

\footnotetext{
* Contribuição técnica ao $69^{\circ}$ Congresso Anual da ABM - Internacional e ao 14ํㅡㄹ ENEMET - Encontro Nacional de Estudantes de Engenharia Metalúrgica, de Materiais e de Minas, 21 a 25 de julho de 2014, São Paulo, SP, Brasil.
} 

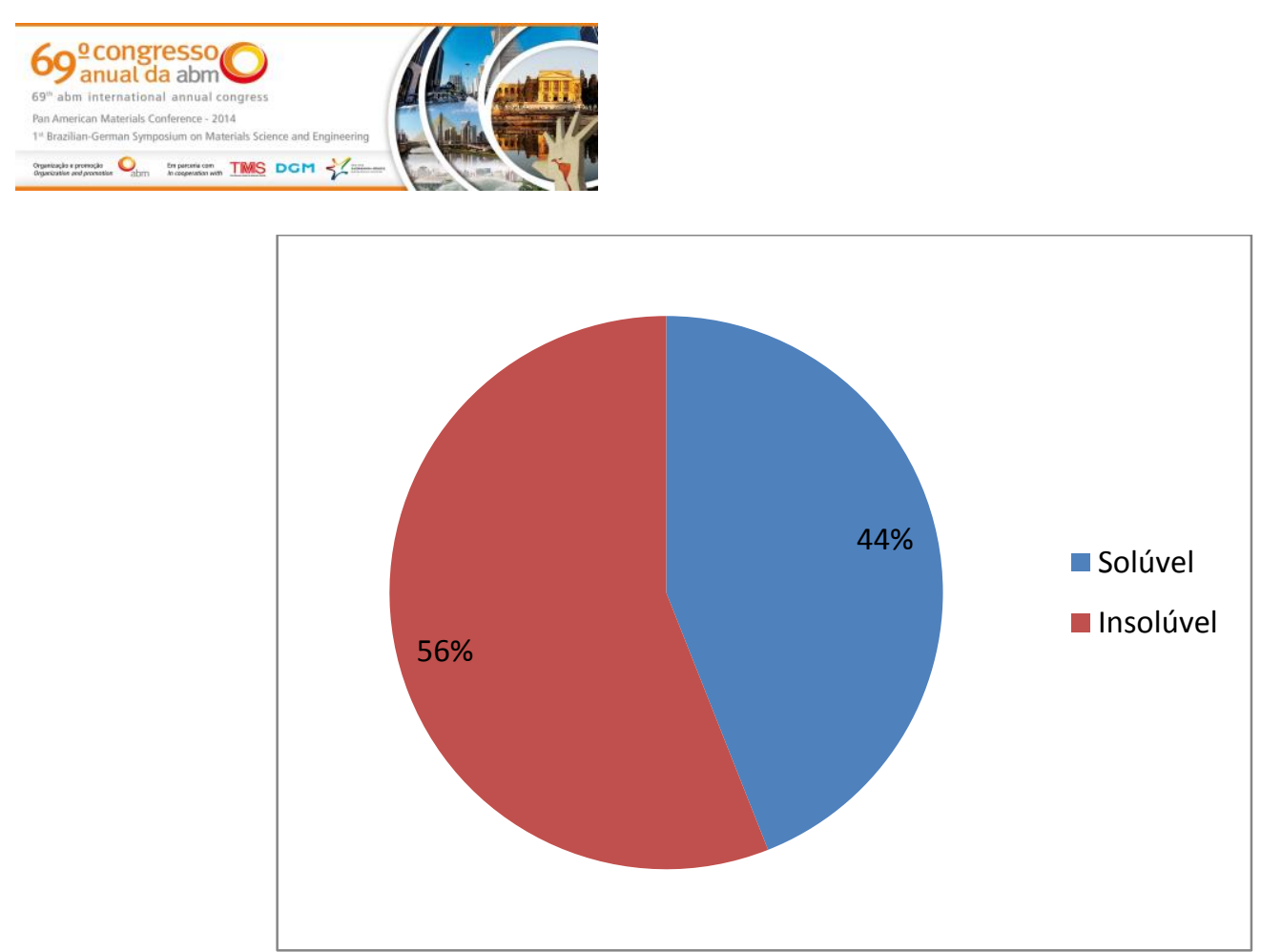

Figura 2. Porcentagem de material magnético e não magnético.

\subsubsection{Espectrometria de emissão óptica com plasma acoplado indutivamente (ICP-OES)}

A alíquota de $10 \mathrm{ml}$ do licor lixiviado foi devidamente diluído e após análise em ICPOES a concentração dos metais presentes na $\mathrm{PCl}$ foi determinada como pode ser visto na Tabela 1.

O cobre é o metal em maior concentração na $\mathrm{PCl}$, representando mais de $22 \%$ de sua composição, seguido do ferro com mais de $7 \%$ e do alumínio com quase $6 \%$.

Considerando somente os metais a quantidade em massa de cobre é superior a soma de todos os outro metais. Isso ocorre devido a alta condutividade do cobre e sua aplicação nas camadas de trilhas da PCI [6].

Tabela 1. Resultados da análise de metais presente no licor lixiviado da água régia

\begin{tabular}{lcc}
\hline Metais & $\begin{array}{c}\% \text { em } \\
\text { massa } \\
\mathrm{PCl}\end{array}$ & $\begin{array}{c}\text { \% em } \\
\text { massa } \\
\text { Metais }\end{array}$ \\
\hline Prata & 0,018 & 0,040 \\
\hline Alumínio & 5,7 & 12,988 \\
\hline Ouro & 0,007 & 0,016 \\
\hline Bário & 0,2 & 0,458 \\
\hline Cálcio & 0,45 & 1,018 \\
\hline Cobre & 22,5 & 51,401 \\
\hline Ferro & 7,3 & 16,745 \\
\hline Níquel & 0,4 & 0,926 \\
\hline Chumbo & 0,03 & 0,063 \\
\hline Estanho & 4,0 & 9,233 \\
\hline Zinco & 3,1 & 7,113 \\
\hline Total & 43,7 & 100,0 \\
\hline
\end{tabular}

Apesar dos metais nobres representarem menos de $1 \%$ da $\mathrm{PCl}$, o valor intrínseco desses metais podem chegar a $80 \%$ do valor da placa [7].

\footnotetext{
* Contribuição técnica ao $69^{\circ}$ Congresso Anual da ABM - Internacional e ao 14ํㅡㄹ ENEMET - Encontro Nacional de Estudantes de Engenharia Metalúrgica, de Materiais e de Minas, 21 a 25 de julho de 2014, São Paulo, SP, Brasil.
} 


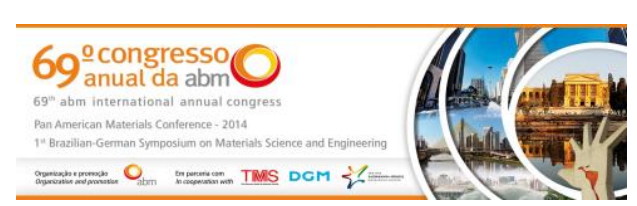

\subsubsection{Perda ao fogo}

O sólido não lixiviado na digestão em água régia, após filtrado e secado passou por ensaio de perda ao fogo. Após ser retirado do forno e pesado o resíduo apresentou perda de massa de $56,7 \%$. Esse valor representa o percentual em massa do sólido não lixiviado volatizado, ou seja, material polimérico. A quantidade de material não volatizado (cerâmico) presente na navícula foi de 43,3\%. Considerando que na digestão em água régia foi identificado que $43,7 \%$ da amostra inicial foi solubilizada, esta parcela corresponde a fração metálica da amostra inicial. Aplicando os percentuais das frações polímeras e cerâmicas identificadas no ensaio de perda ao fogo pode-se afirmar que a $\mathrm{PCl}$ estudada é composta ainda de $31,9 \%$ de polímero e $24,4 \%$ de cerâmicos, conforme Figura 3 . Esses valores correspondem ao verificado por Menetti [6].

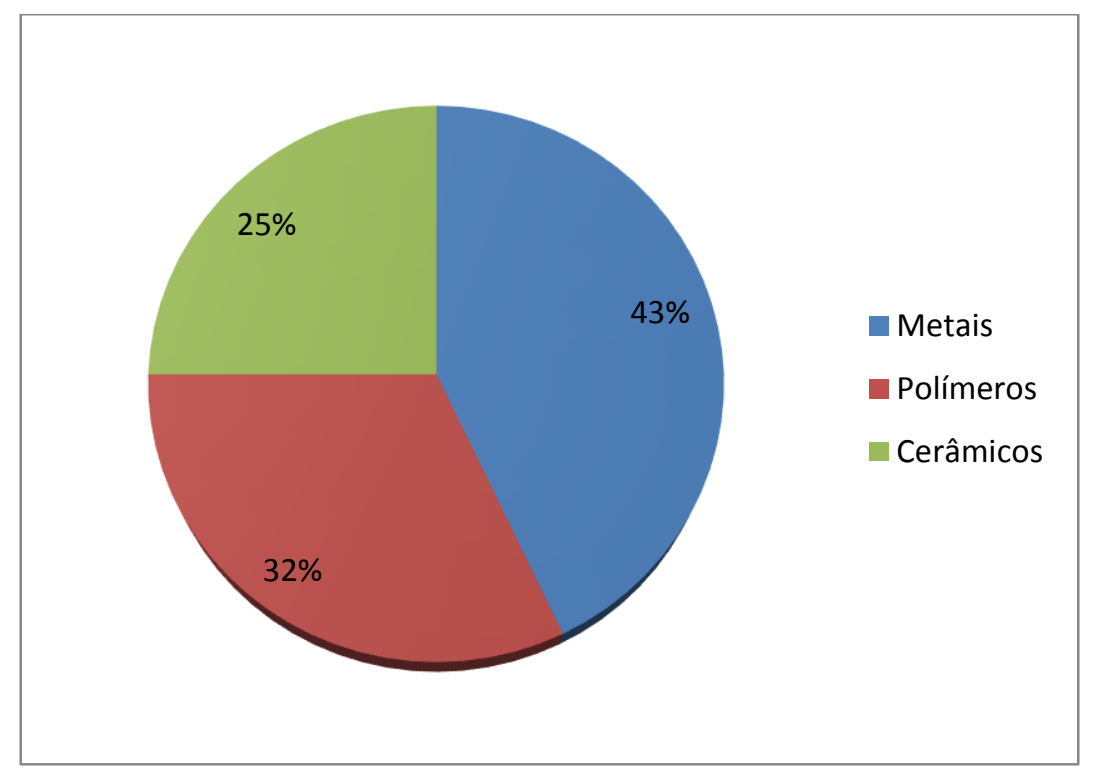

Figura 3. Composição dos materiais da PCl.

Com a identificação de prata nas PCls estima-se que a rota para a reciclagem de nanopartícula de prata pode ser através de moagem, separação magnética, separação eletrostática, extração sólido-liquido, extração liquido-liquido, cristalização e redução.

Sendo que a sintese de nanopartículas de prata pode seguir a rota de redução do nitrato de prata com a adição de citrato de sódio em $39,3 \mathrm{mM}$ e temperatura de $100^{\circ} \mathrm{C}$ com agitação método Turkevitch[16], através da obtenção de extrato lixiviado e purificado contendo nitrato de prata.

As nanopartícula de prata podem ser aplicadas como aditivo para materiais poliméricos, materiais adsorventes de gazes ou metais e ainda em aplicações biotecnológicas.

\section{CONCLUSÃO}

Com o desenvolvimento do trabalho foi possível concluir:

- Do processo de comuinuição somente $1,51 \%$ de perda de materiais.

- As PCls lead free são compostas de aproximadamente $43 \%$ de metais, $25 \%$ de polímeros e $32 \%$ de cerâmicas.

\footnotetext{
* Contribuição técnica ao $69^{\circ}$ Congresso Anual da ABM - Internacional e ao 14ํㅡㄹ ENEMET - Encontro Nacional de Estudantes de Engenharia Metalúrgica, de Materiais e de Minas, 21 a 25 de julho de 2014, São Paulo, SP, Brasil.
} 
